Pre-print Manuscript of Article:

Bridgelall, R., Rafert, J. B., Atwood, D., Tolliver, D., "Hyperspectral Range Imaging for Transportation Systems Evaluation," in Proc. SPIE Smart Structures/NDE 2016, Las Vegas, NV, March 24, 2016.

\title{
Hyperspectral range imaging for transportation systems evaluation
}

\author{
Raj Bridgelall*a ${ }^{*}$ J. Bruce Rafert ${ }^{\mathrm{b}}$, Don Atwood ${ }^{\mathrm{c}}$ and Denver D. Tolliver ${ }^{\mathrm{d}}$
}

\author{
${ }^{a}$ Upper Great Plains Transportation Institute, North Dakota State University, P.O. Box 863676, \\ Plano, TX 75086; ' Department of Physics, North Dakota State University, 1536 Cole Boulevard, \\ Suite 140, Lakewood, CO 80401; ' Michigan Tech Research Institute, 3600 Green Ct., Ann \\ Arbor, MI 48105; ' $U p p e r$ Great Plains Transportation Institute, North Dakota State University, \\ Fargo, ND 58108
}

\begin{abstract}
Transportation agencies expend significant resources to inspect critical infrastructure such as roadways, railways, and pipelines. Regular inspections identify important defects and generate data to forecast maintenance needs. However, cost and practical limitations prevent the scaling of current inspection methods beyond relatively small portions of the network. Consequently, existing approaches fail to discover many high-risk defect formations. Remote sensing techniques offer the potential for more rapid and extensive non-destructive evaluations of the multimodal transportation infrastructure. However, optical occlusions and limitations in the spatial resolution of typical airborne and spaceborne platforms limit their applicability. This research proposes hyperspectral image classification to isolate transportation infrastructure targets for high-resolution photogrammetric analysis. A plenoptic swarm of unmanned aircraft systems will capture images with centimeter-scale spatial resolution, large swaths, and polarization diversity. The light field solution will incorporate structure-from-motion techniques to reconstruct three-dimensional details of the isolated targets from sequences of two-dimensional images. A comparative analysis of existing low-power wireless communications standards suggests an application dependent tradeoff in selecting the best-suited link to coordinate swarming operations. This study further produced a taxonomy of specific roadway and railway defects, distress symptoms, and other anomalies that the proposed plenoptic swarm sensing system would identify and characterize to estimate risk levels.
\end{abstract}

Keywords: 3D reconstruction, hyperspectral image classification, infrastructure performance evaluation, intelligent transportation systems, non-destructive evaluation, photogrammetry, point cloud, structure from motion

\section{INTRODUCTION}

The global transportation network is expansive, dynamic, multimodal, interdependent, and supports the mobility and accessibility needs of a nation. According to data from the Central Intelligence Agency, approximately 40 million miles of roadways, 1.4 million miles of navigable waterways, and more than 40 thousand airports cover the planet's surface. This infrastructure also includes an interacting and interdependent network of railways, pipelines, and shipping ports along with their associated geological and oceanographic structures. Analysts study such complex networks by using models to characterize, understand, and predict their behavior, economics, and risks. The accuracy and utility of those models depend on the quality and timeliness of the inspection data collected to quantify operational performance and to validate condition forecasts. Existing methods of ground-based inspections cannot scale to cover the entire network because of both cost and practical limitations.

The ability of remote sensing methods to capture many image frames with large overlap enables structure-from-motion (SfM) techniques to estimate three-dimensional (3D) measurements from two-dimensional (2D) images. The SfM technique will provide the missing elevation dimension typically required to identify and measure the physical characteristics of a variety of defects in the multimodal transportation infrastructure. Furthermore, combinations of spatial and spectral data will identify uniquely many elements of the global transportation infrastructure that are undetectable or optically occluded in traditional image scenes. Hyperspectral image classification of the spatialspectral data will isolate infrastructure targets from their surroundings to minimize the data volume, thereby reducing overall data storage requirements and processing time. The authors refer to the integration of hyperspectral image 
classification, 3D image reconstruction techniques, and their resulting synergies as hyperspectral structure-frommotion (HSfM). The contributions from this research are to:

1. Review the separate techniques of SfM and hyperspectral image acquisition.

2. Introduce concepts for combining the techniques to derive new utilities from their integration as HSfM.

3. Introduce the enabling platform of plenoptic swarms using unmanned aircraft systems (UAS).

4. Link the utility of HSfM to a taxonomy of applications focused on the non-destructive evaluation (NDE) of multimodal and dynamic transportation infrastructures.

Early applications that fuse hyperspectral data with 3D models tend to utilize techniques such as laser range scanners and LiDAR to recover the 3D coordinates. ${ }^{1}$ Applications that are more recent have developed new workflows to recover the 3D model directly from overlapping hyperspectral images. ${ }^{2}$ The potential for producing high-precision 3D models of the dynamic transportation infrastructure directly from the hyperspectral data will significantly reduce the time, cost, and complexity of image acquisition, processing, and visualization. However, the workflows to reconstruct 3D models seamlessly and automatically from hyperspectral images are still developing. ${ }^{3}$

The organization of this paper begins by reviewing the fundamentals of SfM (section 2.1) and hyperspectral image sensing (section 2.2). Section 2.3 then identifies the synergies of their integration. Those are autonomous UAS navigation to specified targets (section 2.3.1), 3D resolution enhancement (section 2.3.2), and data reduction to increase processing speed (section 2.3.3). Section 2.4 introduces the enabling remote sensing platform as a plenoptic swarm of small UAS (sUAS). The preferred swarming configuration is a multi-resolution pyramid formation (section 2.4.1). Full-frame hyperspectral image sensors will enhance the quality of stitched frames (section 2.4.2) over pushbrooming methods, and existing low-power wireless communications standards (section 2.4.3) will enable practical implementations now. Section 3 produces the taxonomy of specific infrastructure defects that the HSfM technique will identify and characterize. The final section summarizes and concludes the study.

\section{METHODS}

This section reviews the fundamentals and separate utilities of SfM photogrammetry and hyperspectral image classification. The identification of specific methods for their integration highlights the synergies achievable when the process chains become fully automatic and linked to data driven decision-support platforms.

\subsection{Structure from motion and photogrammetry}

The terminology, structure from motion (SfM), refers to an ability to produce three-dimensional (3D) models of objects from sequences of two-dimensional (2D) images. The method has evolved from the field of computer vision, and it is still a subject of considerable research. The SfM techniques offers a lower-cost, lower-power, and less complex alternative to active methods that involve the backscatter sensing of transmitted electromagnetic (EM) waves (e.g. RADAR) and light beams (e.g. LiDAR). ${ }^{4}$ The proliferation of high-resolution consumer grade cameras, freeware, and sUAS platforms has led to a plethora of landscape related applications of photogrammetry. Nevertheless, the generally poor image geometry and resolution achievable from sUAS platforms tend to challenge the performance of SfM algorithms. ${ }^{5}$ For instance, vegetation and steep terrain limits the accuracy of elevation profile estimation. ${ }^{6}$ However, adding spectral information will improve object identification and feature tracking schemes to enhance its overall performance. That is, rather than looking for common tie points in individual bands, the use of hyperspectral permits the identification of target signatures for either exclusion or investigation. For example, the identification of chlorophyll from a spectral signature supports the exclusion of vegetation from SfM processing. The result is a reconstruction of all targets beneath the vegetation in a manner that is similar to the results from foliage penetration (FOPEN) radar.

The overall approach to SfM generally involves several batch operations after the data collection mission. Typical operations include identifying distinct features from the images, ${ }^{7}$ producing their optical flow by matching and tracking correspondence points between images, ${ }^{8}$ estimating the point cloud, ${ }^{9}$ rendering the $3 \mathrm{D}$ visualizations, ${ }^{2}$ and identifying the image scale to extract measurements of the target objects. The SfM algorithms extract a combined rotation and shape matrix that has the following functional decomposition: ${ }^{4}$

$$
W=\left[\begin{array}{llllll}
i_{1}^{T} & \cdots & i_{F}^{T} & j_{1}^{T} & \cdots & j_{F}^{T}
\end{array}\right]^{T}\left[\begin{array}{lll}
p_{1} & \cdots & p_{Q}
\end{array}\right]+t e_{q}^{T} .
$$


The elements of the matrix product are the projections of the 3D points $\boldsymbol{p}$ onto the $(\boldsymbol{i}, \boldsymbol{j})$ coordinate space of the $2 \mathrm{D}$ images $\boldsymbol{W}$. There are $Q$ correspondence points for each of the $F$ frames. The translation matrix is $\boldsymbol{t}$ and $\boldsymbol{e}_{\boldsymbol{q}}$ is the unity matrix. The SfM solution is a singular valued decomposition (SVD) of the $\boldsymbol{W}$ matrix into their separate rotation and shape matrices. Estimating the translation matrix typically involves a simpler algorithm such as averaging the $2 \mathrm{D}$ positions of the correspondence features.

\subsection{Hyperspectral image acquisition}

A hyperspectral scene consists of points $S_{\theta \phi h}$ where the triplet $\{\theta, \phi, h\}$ are the latitude, longitude, and elevation of the geospatial position on the surface $S$. Each point on the surface produces or reflects EM energy with intensity $R_{\Delta \lambda}\left(S_{\theta \phi h}\right.$, $t$ ) where $t$ is the coordinated universal time (UTC) and $\Delta \lambda$ is wavelength span of the EM energy spectrum where

$$
\Delta \lambda=\sum_{i} \lambda[i]
$$

and $\lambda[i]$ is a discrete wavelength band $i$ within the spectral range. Equation (2) holds for light at arbitrary polarization states. Hyperspectral image sensors integrate the EM reflectance from a ground sample area (GSA) into each hyperpixel $p_{x, y, i}$, of the image sensor at time instant $t[n]$ such that:

$$
p_{x, y, i}[n]=R_{\lambda[i]}\left(G_{s}[x, y], t[n]\right)
$$

$G_{s}[x, y]$ is the center of the GSA with dimensions $G_{x} \times G_{y}$ meters. Practitioners generally refer to the $G_{x}$ dimension as the ground sampling distance (GSD).

\subsection{Synergy of methods}

This section identifies the main synergies achieved from the combination of the SfM and hyperspectral approaches. References to previous work by the authors provide details that benchmark their relative performance as a function of classification accuracy and computational complexity.

\subsubsection{Rapid hyperspectral image classification for real-time navigation}

With sufficient spectral resolution and span, the spectral shape and reflectance levels of each hyper-pixel forms a unique signature that enhances the ability of classification algorithms to identify unique objects in noisy and low spatial resolution images. ${ }^{11}$ The computational complexity of typical image classification methods precludes the realtime identification of landscape elements by utilizing the computing capacity of energy-efficient mobile platforms. However, the authors have recently developed techniques of rapid hyperspectral image classification, such as the simple spectral classifier (SSC), that would enable the autonomous navigation of sUAS along transportation corridors such as roadways, railways, pipelines, and waterways. ${ }^{12}$ Real-time classification of the landscape provides accuracy indicators to achieve resolution agile remote sensing. ${ }^{13}$ That is, the aircraft could fly closer to potential targets until the spatial resolution is sufficient to yield the desired threshold of classification accuracy, and then autonomously return to the originally programmed flight path.

\subsubsection{Occlusion removal and resolution enhancement}

In the visible spectrum, portions of an object may appear at some camera positions and be obscured at others. Hyperspectral images will extend the information in typical point clouds because each pixel in Equations (1) and (3) contains information that could identify portions of the target object that remain obscured in the visible spectrum. The corresponding hyperspectral and polarization data at other camera positions could identify and remove materials that camouflage portions of the object in those frames. Subsequently, the fusion of SfM operations from the individual spectral bands, or alternatively, producing the optical flow of correspondence points by tracking their complete hyperspectral signatures will produce additional target pixels that result in denser point clouds. Previous studies found that adding spectral data from only six channels of a hyperspectral scene can increase the number of correspondence points over standard images by a factor of more than six. ${ }^{3}$ Higher density point clouds improve the accuracy and precision of geometric measurements extracted from the mesh reconstructions. ${ }^{10}$

\subsubsection{Target isolation and data reduction}

Hyperspectral image classification will also identify non-target objects that the process can eliminate from SfM reconstruction. The removal of spurious pixels will substantially reduce the computational time and storage requirements. Rapid classification of hyperspectral scenes would enable real-time data reduction for either on-board 
or offline processing of specific targets. This synergy of approaches has the potential to produce complete highresolution $3 \mathrm{D}$ visualizations and measurements of defects on isolated transportation infrastructure such as roads, bridges, railways, and pipelines.

\subsection{Multiresolution remote sensing platform}

This section introduces the platform concept to enable simultaneous multiresolution remote sensing. The ground sampling distance (GSD) is the lateral distance $G_{x}$ on the ground that reflects or emits EM energy into each hyperpixel of lateral dimension $c_{x}$ such that

$$
G_{x}=c_{x} M_{\eta}^{1 / 2}
$$

where $M_{\eta}$ is the optical magnification factor of the sensor. ${ }^{11}$ Therefore, an arbitrary factor reduction in the effective pixel size will result in the same factor reduction in the GSD. For a fixed instantaneous field-of-view (IFOV) camera and flight altitude $h_{s}$ the GSD is

$$
G_{x}=2 h_{s} \tan \left(\frac{I F O V}{2}\right)
$$

For small angles, $\tan (\theta) \approx \theta$, hence $G_{x} \approx h_{s} \times$ IFOV. Previous work established that centimeter-scale depth resolution is achievable from flight altitudes of approximately 30 meters using a standard fix-focus color camera with pixel dimensions of 4.88 micrometers. ${ }^{14}$ In practice, however, objects such as trees, utility poles, signage trestles, and other tall structures could preclude flying at altitudes lower than a few meters. Adaptive zooming may enable higher spatial resolution at higher altitudes, ${ }^{15}$ but researchers have established that fixed-focus cameras produce the best image sources for photogrammetric processing. ${ }^{16}$ Static focus is also preferred for capturing dynamic events such as moving traffic.

\subsubsection{Plenoptic unmanned aircraft swarm configuration}

Figure 1 introduces the plenoptic pyramid swarm (PPS), which is one of many possible configurations.

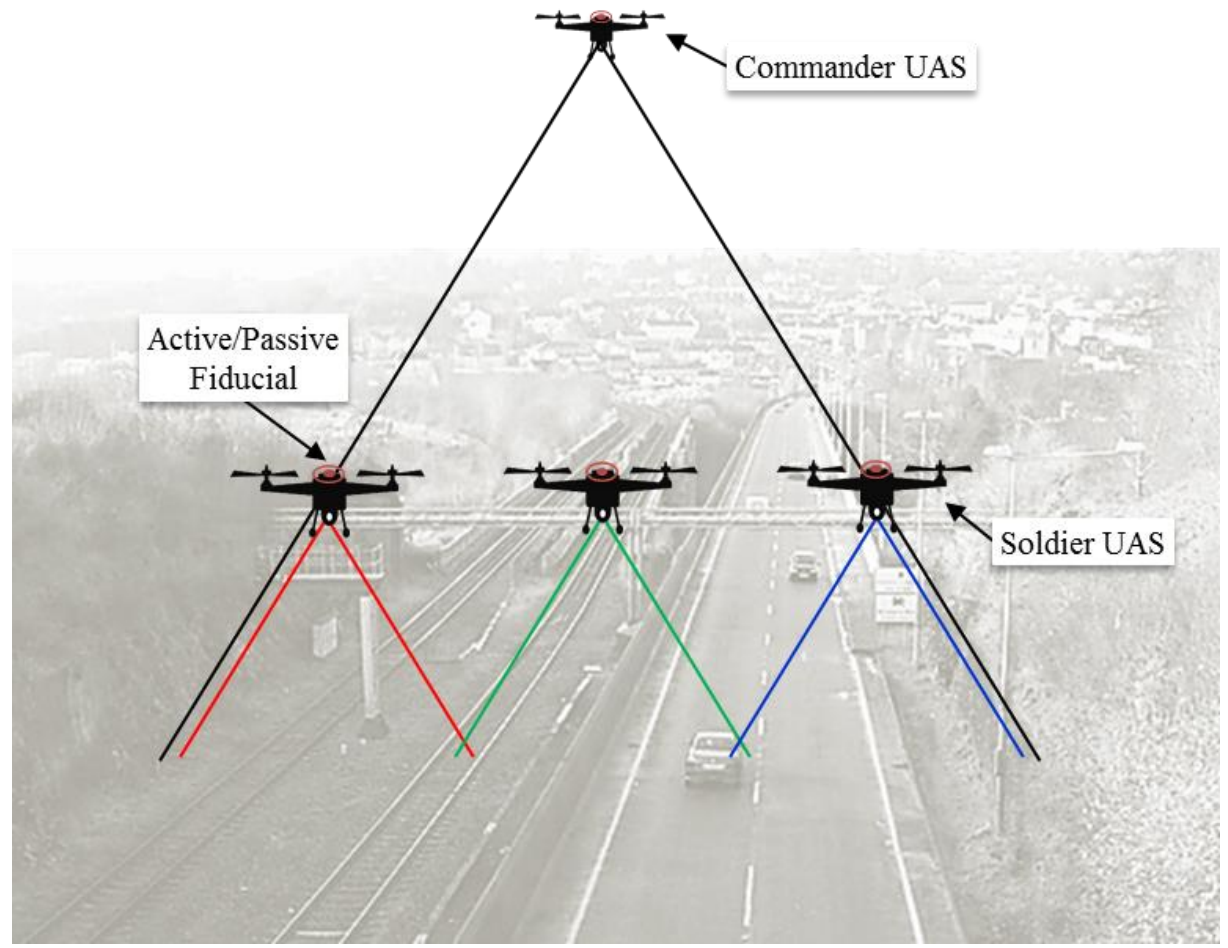

Figure 1. Multiresolution hyperspectral plenoptic pyramid swarm.

The generalized arrangement is as a multi-layer pyramid that in aggregate forms a multiresolution light field camera. Each platform carries a fixed focus hyperspectral camera arranged to provide the desired IFOV overlap for image stitching, angular diversity to improve stereoscopic methods of elevation information recovery, and polarization 
diversity to mitigate potential issues of specular reflections. Combining the narrower IFOV from multiple parallel platforms will provide both the higher spatial resolution and swath coverage desired. A commander UAS occupies the highest altitude to provide real-time and autonomous navigation by pre-classifying the scene within a larger IFOV. The lower spatial resolution images from the commander UAS will be sufficient to enable rapid object recognition so that the aircraft can autonomously identify and follow any portion of the railroad tracks or the roadway that deviates from any pre-programmed mission coordinates. The aggregate scene from a collection of soldier UAS will provide the higher spatial resolution needed for NDE.

The image stitching algorithms will use the field-of-view from the commander UAS that includes the under-flying soldier UAS to improve the efficiency and accuracy of scene reconstruction and orthographic rectification. Depending on the requirements, configuration, and data acquisition speed for the specific monitoring application, the swarmsensing configuration illustrated in Figure 1 is extensible to include multiple layers, additional lateral and longitudinal coverage for greater angular and/or polarization diversity. Other formations that could require dozens of sUAS are also likely to provide greater visibility under noisy conditions.

\subsubsection{Full-frame hyperspectral image assembly}

The low spatial resolution of hyperspectral images available from traditional sources such as high-altitude airborne and spaceborne platforms has historically limited the utility of hyperspectral image classification in the NDE of transportation infrastructure. ${ }^{11}$ However, the proliferation and cost reduction of monolithic image sensors and sUAS platforms has presented new opportunities. ${ }^{12}$ The agility of rotary sUAS improves accessibility to monitor large structures such as bridges, overpasses, and intermodal facilities. Nevertheless, the inherent attitude instability of rotary sUAS from wind perturbations increases the difficulty of orthographically correcting and assembling complete scenes from pushbrooming sources. ${ }^{17}$ Fortuitously, the emergences of compact lightweight cameras that provide full-frame hyperspectral images change that paradigm. ${ }^{18}$ The additional frame overlap that full-frame hyperspectral imagers can provide over line scanning via the pushbrooming method will increase the number of trackable correspondence points. ${ }^{19}$ Subsequently, full-frame images result in less distortion after frame stitching and orthographic rectification. ${ }^{20}$

The implementation of full-frame hyperspectral imagers typically involves a trade-off that decreases the spatial resolution over line scanners. For example, an implementation that uses mosaic configurations such as $5 \times 5$ pixel arrays provides 25 spectral channels per hyper-pixel. ${ }^{21}$ Therefore, the hyper-pixels are 5-times larger than the native pixels of the image sensor array. For a fixed flight altitude and optical camera geometry, the larger effective pixel sizes reduce the spatial resolution achievable with an equivalent color imager. To compensate for the loss in spatial resolution relative to pushbrooming methods, a platform using full-frame hyperspectral sensors must decrease its altitude or decrease its IFOV by a factor equal to the hyper-pixel array dimension. Alternatively, the design could distribute the reduction factor between the altitude and IFOV. Decreasing the IFOV has the advantage of decreasing geometric distortions that hamper the identification of targets but the disadvantage of lowering the swath coverage. ${ }^{22}$

\subsubsection{Communications and control strategy}

The vertical separation distance between commander and soldier UAS with identical IFOV will be at least $N_{u} \times h_{s}$ where $N_{u}$ is the number of soldier UAS per commander UAS. For a highway lane-scanning scenario, Equation (5) determines that a single soldier sUAS carrying a camera with an IFOV of 18-degrees must fly at an altitude of 11.5meters ( $\sim 38$-ft) to provide the swath distance needed to cover a typical 12-ft wide lane. Hence, a 4-lane highway will require at least four sUAS plus one additional unit to include coverage for the road median and shoulders. Therefore, the commander sUAS must fly at an altitude of approximately 58-meters ( 190-ft) to guide the five-fleet sUAS layer along the highway corridor. The actual GSD needed to discern and evaluate specific features of the transportation infrastructure, and the camera parameters (hyper-pixel size and IFOV) will determine the actual flight altitude for the soldier sUAS, and in turn the commander sUAS. This scenario provides some insights as to the typical communications distance requirement between soldier and commander sUAS.

A wide variety of media (light, ultrasonic, radio waves) is available for implementing a low-power wireless communications system for devices in motion. Practitioners tend to use the radio frequency media because of their robustness in noisy environments, long-range with low power consumption, omnidirectional properties, compactness of available implementations, and ease of integration into lightweight mobile platforms. Numerous types of affordable, ultra-low-power (tens of microwatts) and standards compliant electronic modules are commercially available for integration into sUAS. Figure 2 classifies the available radio frequency standards in terms of their power supply and communications media, and summarizes the key performance tradeoffs. ${ }^{23}$ 
The active far-field systems are most appropriate for implementing the PPS formation. Within that subset, the Wi-Fiß and Bluetooth ${ }^{\circledR}$ standards lack the range and rapid association capabilities needed to maintain an agile swarm configuration. The ultra-high-frequency (UHF) bands provide license-free and robust communications well beyond one kilometer. The data rates, however, are low relative to $\mathrm{Wi}-\mathrm{Fi}{ }^{\circledR}$ and Bluetooth ${ }^{\circledR}$ standards. The UHF data rates can support small packets of command and control data to enable the swarming formation. ${ }^{24}$ The expected design will require that each packet identify the sUAS uniquely, accommodate a few navigational control commands, and include encryption information for secure communications. Therefore, the final selection of wireless communications standard will depend on the swarm configuration, flight altitude, aircraft maneuverability, top speeds, and the amount of information that each aircraft must exchange to maintain a safe and reliable navigation.

\begin{tabular}{|c|c|c|c|c|}
\hline & \multicolumn{2}{|c|}{ Near-Field } & \multicolumn{2}{|c|}{ Far-Field } \\
\hline & \multicolumn{2}{|c|}{ e.g. RuBee (IEEE P1902.1), NFC (ISO 18092) } & \multicolumn{2}{|c|}{ e.g. Wi-Fi®, Bluetooth®, UHF (ISO18000-7) } \\
\hline 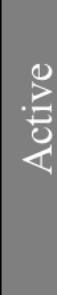 & $\begin{array}{l}\text { Benefits } \\
\text { - Robust link around } \\
\text { dense RF media } \\
\text { - Magnetic field zone } \\
\text { control } \\
\text { - Simple narrow-band } \\
\text { protocols maximize } \\
\text { battery life }\end{array}$ & $\begin{array}{l}\text { Deficiencies } \\
\text { - Range limited to antenna } \\
\text { loop diameter } \\
\text { - Multi-node arbitration } \\
\text { speed limited by data } \\
\text { rate }\end{array}$ & $\begin{array}{l}\text { Benefits } \\
\text { - 100s of meter range } \\
\text { from RF propagation \& } \\
\text { high transmit power } \\
\text { - High multi-node } \\
\text { arbitration rates possible } \\
\text { due to larger bandwidth } \\
\text { \& data-rate }\end{array}$ & $\begin{array}{l}\text { Deficiencies } \\
\text { - Some bands require } \\
\text { spread spectrum \& } \\
\text { complex multiple access } \\
\text { protocols; leads to } \\
\text { higher power } \\
\text { consumption } \\
\text { - Poor zone control }\end{array}$ \\
\hline & \multicolumn{2}{|c|}{ e.g. HF (ISO 14443), LF (ISO 14223-1) } & \multicolumn{2}{|c|}{ e.g. UHF (ISO18000-6) } \\
\hline 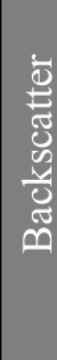 & $\begin{array}{l}\text { Benefits } \\
\text { - Excellent zone control } \\
\text { - Robust near-field energy } \\
\text { harvesting Robust media } \\
\text { penetration }\end{array}$ & $\begin{array}{l}\text { Deficiencies } \\
\text { - Backscatter sensor } \\
\text { sensitivity \& loop } \\
\text { antenna diameter limits } \\
\text { practical range to within } \\
\text { one meter } \\
\text { - Multi-node arbitration } \\
\text { limited by bandwidth \& } \\
\text { data rate }\end{array}$ & $\begin{array}{l}\text { Benefits } \\
\text { - Tens of meters of range } \\
\text { for passive RFID } \\
\text { - Longer range for semi- } \\
\text { passive RFID; limited } \\
\text { primarily by reader } \\
\text { sensitivity } \\
\text { - High multi-node } \\
\text { arbitration rate } \\
\text { - Longer battery life }\end{array}$ & $\begin{array}{l}\text { Deficiencies } \\
\text { - Poor zone control } \\
\text { - Poor RF media } \\
\text { penetration } \\
\text { - Highly orientation } \\
\text { sensitivity due to } \\
\text { weaker backscatter and } \\
\text { multi-path propagation }\end{array}$ \\
\hline
\end{tabular}

Figure 2. Classification of potential candidates for the ultra-low-power wireless communications standard.

For a PPS scenario, the commander sUAS will instruct soldier sUAS to maintain a predetermined and fixed flight altitude below it. The exchange of global positioning system (GPS) coordinates and inertial data will enable the commander sUAS to maintain the desired position of each soldier sUAS. Active and/or passive fiducials will allow the commander sUAS to fine-tune and maintain soldier sUAS alignment beneath it, irrespective of GPS accuracy. Active fiducials may consist of flashing infrared lights whereas passive fiducials may consist of high reflectivity materials that have unique spectral signatures and/or shapes for easy identification. Monolithic systems-on-a-chip solutions are available to identify unique shapes such as a bullseye or a barcode pattern at high speeds and under a broad range of noise and affine distortions. ${ }^{25}$

This research does not address the endurance limitation of sUAS. However, future research will describe emerging techniques that utilize charging and launching road vehicles that could alternate fully charged fleets and reposition themselves at the appropriate landing sites.

\section{DISCUSSION}

Practitioners identify distress symptoms of transportation infrastructure by measuring centimeter-scale anomalies to assess and quantify their condition. ${ }^{26}$ Roadway anomalies that can pose hazards to the traveling public include potholes, frost heaves, and concrete pavement upheavals. Distress symptoms such as rutting, cracking, settlement, and 
crown loss are key factors that determine the remaining service life of pavements. The current approaches that include manual inspections and surface profiling equipment cannot scale to provide network wide assessments because of cost and practical limitations.

Railroads must visually inspect most tracks in service as often as twice weekly to comply with the federal track safety standards. Typical faults include the misalignment of rail geometry, broken and worn track components, deteriorating support structures, and track obstructions. The rate of defect formation increases with the load density of traffic, hence railroads are seeking rapid and frequent means of analyzing and detecting faults that can result in accidents, delays, lost revenue, and tarnished brands. In addition to visual inspections, current methods to locate developing and mature defects involve rail inspection cars that are equipped with a variety of NDE sensors such as ultrasonic, LiDAR, eddy current, and high-illumination imagers. However, such methods are relatively slow and they cannot detect all faults. ${ }^{27}$ Furthermore, track inspections require closures that result in a loss of line capacity and diminished revenue service.

The assessment of typical faults and distress symptoms that the HSfM method will identify and quantify (Figure 3) stems from the authors many years of collective experiences in the field. Although the method is applicable to all types of transportation infrastructure, the assessment focuses on roadways and railways because they carry the majority of freight and passengers.
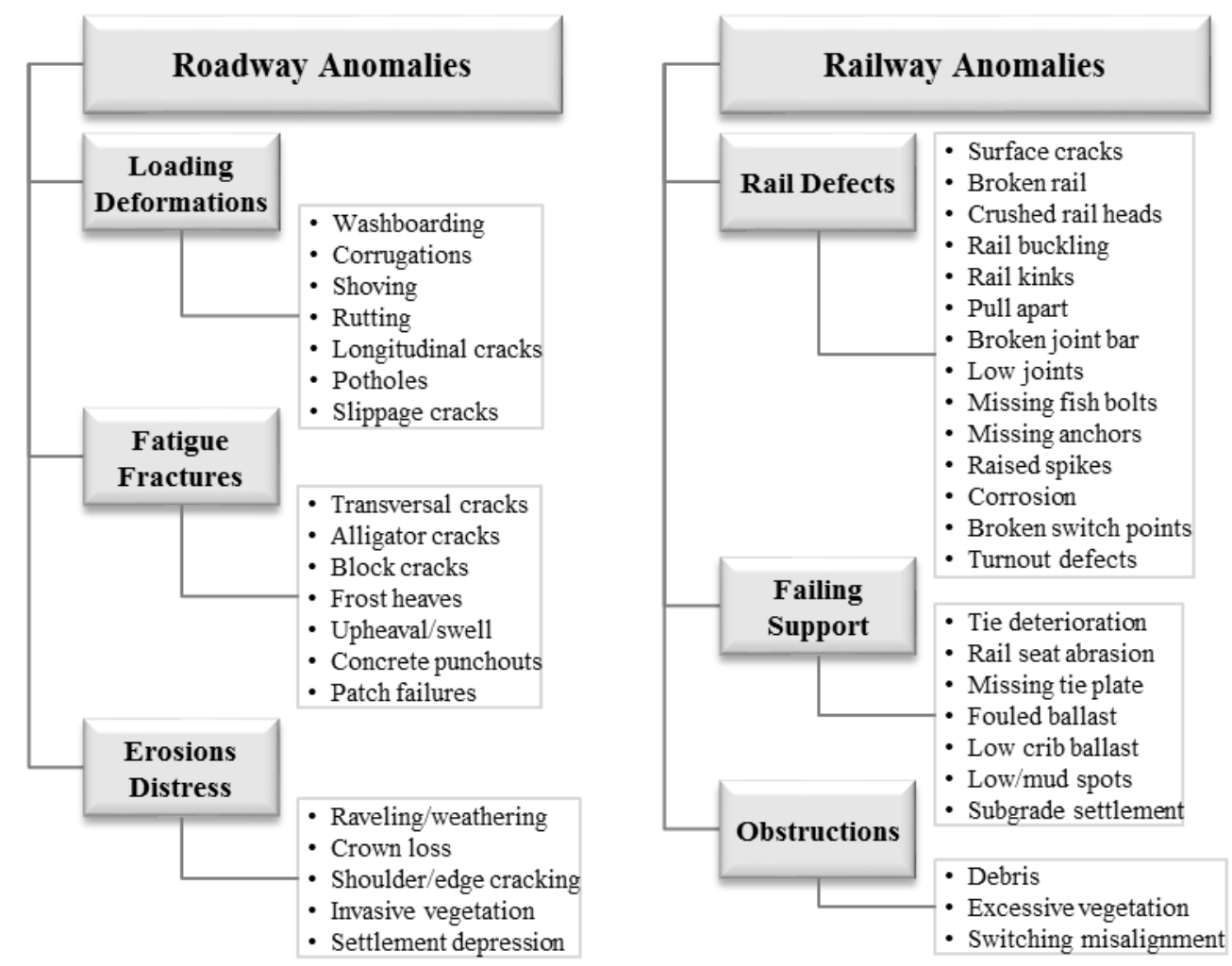

Figure 3. Roadway and railway anomalies that require sub-centimeter level spatial resolution to characterize.

Related work validates this assessment under different levels of constraints. In particular, researchers have determined that it is possible to measure the vertical dimension of potholes, washboarding, and crown loss with centimeter-level accuracy when using standard color imagers aboard sUAS. ${ }^{14}$ Research in adjacent fields found that the precision of measuring plant heights of one-meter is greater than $10 \%$ when using color images acquired at a spatial resolution of $5-\mathrm{cm}$ and $80 \%$ frame overlap from a fixed wing unmanned aerial vehicle (UAV). ${ }^{28}$ Those results provide confidence that synergies resulting from the HSfM approach and the use of multiresolution swarm sensing platforms will yield the anticipated results. 


\section{SUMMARY AND CONCLUSIONS}

Existing methods of transportation infrastructure NDE that uses ground patrol equipment and conventional remote sensing techniques cannot scale to provide cost-effective solutions. This research proposes a new Hyperspectral Structure-from-Motion (HSfM) paradigm that scales cost-effectively. The enabling remote sensing platform is a swarm configuration of low-cost sUAS that implements a multiresolution plenoptic imaging system that has the capacity to cover vast areas in short periods. Rapid hyperspectral image classification will enable autonomous swarm navigation to achieve a multiresolution capability that provides high-resolution images of selected areas that contain potential targets. SfM algorithms will achieve higher accuracy 3D measurements by using the additional correspondence points available from hyperspectral pixels. The enhanced material identification capability of hyperspectral image classification will eliminate non-transportation targets to reduce the data volume and increase the SfM processing speeds. A classification and assessment of commercially available low-cost wireless communications standards suggests that the best-suited implementation to synchronize the swarm sensing operations will be application dependent.

Transportation systems adapt continually to changes in demand and to the deployment of new technologies. The authors leveraged their extensive experience in the practical aspects of transportation infrastructure NDE to produce a taxonomy of the anomaly types that the HSfM solution will identify and characterize. The HSfM paradigm offers adaptability and scalability to support evolving NDE needs. Future research will assess the tradeoff in accuracy and precision of detecting a catalog of target anomalies by using a fleet of commercially available sUAS, full-frame hyperspectral imagers, 3D modeling software, and custom software to extract the measurements. The authors plan to integrate the HSfM workflows in dynamic data driven applications systems that enable a variety of decision-support.

\section{ACKNOWLEDGEMENT}

A grant from the Mountain Plains Consortium supported this research.

\section{REFERENCES}

[1] Kim, M. H., Harvey, T. A., Kittle, D. S., Rushmeier, H., Dorsey, J., Prum, R. O., and Brady, D. J., "3D Imaging Spectroscopy for Measuring Hyperspectral Patterns on Solid Objects," ACM T Graphic, 31(4), 1-11 (2012).

[2] Zia, A., Liang, J., Zhou, J., and Gao, Y., "3D Reconstruction from Hyperspectral Images," Proc. WACV 2015, 318-325, (2015).

[3] Miller, C. A., and Walls, T. J., "Passive 3D Scene Reconstruction via Hyperspectral Imagery," [Advances in Visual Computing], Springer International Publishing, New York, 413-422 (2014).

[4] Shah, M., and Jain, R., Eds., [Motion-based Recognition Volume 9], Springer Science and Business Media, New York (2013).

[5] Micheletti, N., Chandler, J. H., and Lane, S. N., "Structure from Motion (SfM) Photogrammetry," [Geomorphological Techniques], L. Clarke and J. Nield, Eds., British Society for Geomorphology, London (2015).

[6] Tonkin, T. N., Midgley, N. G., Graham, D. J., and Labadz, J. C., "The Potential of Small Unmanned Aircraft Systems and Structure-from-Motion for Topographic Surveys: A Test of Emerging Integrated Approaches at Cwm Idwal, North Wales," Geomorphology, 226, 35-43 (2014).

[7] Lowe, D. G., "Distinctive Image Features from Scale-Invariant Keypoints," Int J Comput Vision, 60(2), 91 110 (2004).

[8] Lucas, B. D., and Kanade, T., "An Iterative Image Registration Technique with an Application to Stereo Vision," Proc. IJCAI 1981, 674-679 (1981). 
[9] Seeger, M., Gronz, O., Klaes, B., Becker, K., Marzen, M., and Remke, A., "The Effect of Object Characteristics and Image Quality on 3D-Modelling by Structure-from-Motion," EGU2014-7293, (2014).

[10] Arikan, M., Preiner, R., Scheiblauer, C., Jeschke, S., and Wimmer, M., "Large-Scale Point-Cloud Visualization Through Localized Textured Surface Reconstruction," IEEE T Vis Comput Gr, 20(9), 12801292 (2014).

[11] Bridgelall, R., Rafert, J. B., and Tolliver, D. D., "Hyperspectral Imaging Utility for Transportation Systems," Proc. SPIE 9435, 943522 (2015).

[12] Bridgelall, R., Rafert, J. B., \& Tolliver, D. D., "Emerging Applications of Hyperspectral Image Sensing in Transportation," Sensors Expo and Conference 2015, (2015).

[13] Bridgelall, R., Rafert, J. B., Tolliver, D. D., and Lee, E., "Resolution agile remote sensing for hazardous material spill detection," TRB 2016, (2016).

[14] Dobson, R. J., Colling, T., Brooks, C., Roussi, C., Watkins, M. K., and Dean, D., "Collecting Decision Support System Data Through Remote Sensing of Unpaved Roads," Transport Res Rec, 2433, 108-115 (2014).

[15] Enoc, S.-A., Chandler, J. H., and Rene, W., "Parametrising Internal Camera Geometry with Focusing Distance," Photogramm Rec, 27(138), 210-226 (2012).

[16] Shortis, M. R., Bellman, C. J., Robson, S., Johnston, G. J., and Johnson, G. W., "Stability of Zoom and Fixed Lenses used with Digital SLR Cameras," Proc. ISPRS XXXVI, 5, 25-27 (2006).

[17] Nackaerts, K., Delauré, B., Everaerts, J., Michiels, B., Holmlund, C., Mäkynen, J., and Saari, H., "Evaluation of a Lightweight UAS-Prototype for Hyperspectral Imaging," Proc. ISPRS XXXVIII, 5, 478-483 (2010).

[18] Bareth, G., Aasen, H., Bendig, J., Gnyp, M. L., Bolten, A., Jung, A., Michels, R., and J. Soukkamäki, "LowWeight and UAV-Based Hyperspectral Full-Frame Cameras for Monitoring Crops: Spectral Comparison with Portable Spectroradiometer Measurements," Photogramm Fernerkun, 2015(1), 69-79 (2015).

[19] Micheletti, N., Chandler, J. H., and Lane, S. N., "Investigating the Geomorphological Potential of Freely Available and Accessible Structure-from-Motion Photogrammetry using a Smartphone," Earth Surf Proc Land, 40(4), 473-486 (2014).

[20] Aasen, H., Bendig, J., Bolten, A., Bennertz, S., Willkomm, M., \& Bareth, G., "Introduction and Preliminary Results of a Calibration for Full-Frame Hyperspectral Cameras to Monitor Agricultural Crops With UAVs," Proc. ISPRS XL-7, 1-8 (2014).

[21] Geelen, B., Tack, N., and Lambrechts, A., "A Compact Snapshot Multispectral Imager with a Monolithically Integrated Per-Pixel Filter Mosaic," Proc. SPIE 8974, 89740L (2014).

[22] Madden, M., Jordan, T., Bernardes, S., Cotten, D. L., O’Hare, N., and Pasqua, A., "Unmanned Aerial Systems and Structure from Motion Revolutionize Wetlands Mapping," [Remote Sensing of Wetlands: Applications and Advances], R. W. Tiner, M. W. Lang and V. V. Klemas, Eds., CRC Press, Boca Raton, 195-219 (2015).

[23] Bridgelall, R., "Introducing a Micro-wireless Architecture for Business Activity Sensing," Proc. IEEE CRFID 2008, (2008).

[24] Bridgelall, R., "System and a Node used in the System for Wireless Communication and Sensory Monitoring," United States of America Patent 7,433,648 (2008).

[25] Carlson, B., and Bridgelall, R., "Imager Integrated CMOS Circuit Chip and Associated Optical Code Reading Systems," United States of America Patent 11/879,051 (2007).

[26] Bridgelall, R., "Precision Bounds of Pavement Distress Localization with Connected Vehicle Sensors," J Infrastruct Syst, 21(1), 04014033 (2015).

[27] Bridgelall, R., "RAILCOTS -- Rolling Stock Automatic In-Situ Line-Quality, Car Operations and Tracking System," Sensors Expo and Conference 2013, (2013). 
Hyperspectral Range Imaging for Transportation Systems Evaluation

[28] Zarco-Tejada, P. J., Diaz-Varela, R., Angileri, V., and Loudjani, P., "Tree Height Quantification using Very High Resolution Imagery Acquired from an Unmanned Aerial Vehicle (UAV) and Automatic 3D PhotoReconstruction Methods," Eur J Agron, 55, 89-99 (2014). 